\title{
THz-SAR Vibrating Target Imaging via the Bayesian Method
}

\author{
Bin Deng, Xin-yun Wang, Cheng-guang Wu, Yu-liang Qin, and Hong-qiang Wang \\ College of Electronic Science and Engineering, National University of Defense Technology, 157 Deya Road, Changsha 410073, China \\ Correspondence should be addressed to Bin Deng; sagitdeng@163.com
}

Received 18 September 2016; Revised 7 January 2017; Accepted 22 January 2017; Published 19 February 2017

Academic Editor: Lorenzo Crocco

Copyright (C) 2017 Bin Deng et al. This is an open access article distributed under the Creative Commons Attribution License, which permits unrestricted use, distribution, and reproduction in any medium, provided the original work is properly cited.

\begin{abstract}
Target vibration bears important information for target recognition, and terahertz, due to significant micro-Doppler effects, has strong advantages for remotely sensing vibrations. In this paper, the imaging characteristics of vibrating targets with THz-SAR are at first analyzed. An improved algorithm based on an excellent Bayesian approach, that is, the expansion-compression variancecomponent $(\mathrm{ExCoV})$ method, has been proposed for reconstructing scattering coefficients of vibrating targets, which provides more robust and efficient initialization and overcomes the deficiencies of sidelobes as well as artifacts arising from the traditional correlation method. A real vibration measurement experiment of idle cars was performed to validate the range model. Simulated SAR data of vibrating targets and a tank model in a real background in $220 \mathrm{GHz}$ show good performance at low SNR. Rapidly evolving high-power terahertz devices will offer viable THz-SAR application at a distance of several kilometers.
\end{abstract}

\section{Introduction}

Vibration is one of the commonest motions in nature, such as the vibration of a car engine, the bumping of a car on a rude road, the chest movement caused by heart beat and breath, and the arm swing of a walking terrorist. The characteristics of vibration differ greatly for different targets. For example, the vibration amplitude of different types of tanks or armored cars ranges from several millimeters to several centimeters, and the vibration frequency ranges from several hertz to several decades hertz. The exact values are closely related to the vehicle type, size, and structure. In addition, the remote sensing of vibration is helpful for gathering the road condition information [1]. One can see that vibration contains subtle and stable information of targets. The vibration parameters, once extracted, can play an important role in target recognition, which will open up a new range of applications in battlefield reconnaissance, precision seeking, traffic surveillance, and antiterrorist tasks.

Detecting vibrating targets on the ground with synthetic aperture radar (SAR) is an emerging subject in the remote sensing community. Direct SAR imaging for vibrating targets, however, will result in ghosts in the image due to the periodic micro-Doppler modulation [2], which cannot be overcome by the SAR ground moving target indication (SAR/GMTI) techniques. Relevant researches on SAR target vibration have been carried out internationally since 1995 [3], and our group started the study early in 2006 in China and defined it, together with rotating target detection and imaging, as SAR/micromotion target indication (SAR/MMTI) [4]. Recent works on it are mainly focused on the vibrating target phenomenology or vibration's influence, vibrating target detection, micro-Doppler analysis as well as extraction, and real vibration sensing experiments. On the phenomenology, main conclusion is that vibration gives rise to high frequency phase and paired echoes in the time domain, to micro-Doppler in the frequency domain and to ghosts [2], fence [5], bowknot [6], or fuzzballs [7] in the image domain. On the detection, the main methods include the generalized likelihood ratio test and cyclostationary spectrum density method [8-10] or the multiantenna based displaced phase center antenna (DPCA) and along-track interferometry (ATI) methods [11-14]. On the micro-Doppler analysis and extraction, time-frequency analysis [12] and the fractional Fourier transform (FrFT) [15] are predominant and the pulse-repetition-interval transform [16] can also provide good results. On the experiments, X-band APY-6 [17], Kuband Lynx [18] and mmW MEMPHIS [12] radar systems have been utilized. The basic viability of vibration sensing has been proven, but two main problems have yet to be addressed. (1) Experimental data show that minor vibrations with the amplitude under several millimeters cannot be detected even 
by mmW radars [12]. (2) Few researches on vibrating target imaging have been done. Although the phase compensation method and the Doppler keystone transform [19] can be used for imaging, they are mainly useful to point scattering targets, and the range cell migration of vibrating targets is still difficult to be corrected. The MLE imaging method [20] proposed previously by us can directly model the echo signal and then estimate scattering centers of vibrating targets but is essentially of the high-dimensional nonlinear optimization problem with ill condition, and it is impossible to obtain the global minimum without using prior information.

To solve problem (1), we introduce terahertz wave into SAR for vibration sensing. Due to the shorter wavelength, terahertz wave is more sensitive to micromotions, making it easier to detect vibrating targets. The emission power of solidstate and vacuum electronics terahertz devices approaches to several decade Watts according to the DARPA terahertz electronics program. As there is serious atmosphere attenuation, the emission power is sufficient to meet the needs of airborne terahertz radar for operating range of several kilometers at the frequencies of $220 \mathrm{GHz}$ or $330 \mathrm{GHz}$ atmosphere windows. In 2012, the US DARPA proposed a project called Video SAR (ViSAR) [21] and the frequency band around $230 \mathrm{GHz}$ was chosen, prophesying realization of THz-SAR. To solve problem (2), we convert vibrating target imaging to the problem of sparse representation, and the expansion-compression variance-component $(\mathrm{ExCoV})$ method is utilized to obtain the scattering coefficients of vibrating targets. The ExCoV method is of sparse Bayesian learning (SBL) [22], but it is more effective and has less parameters than the traditional SBL and is promising to efficiently solve the high-dimensional optimization problems. In this paper, we will modify the initial iteration in ExCoV, that is, superseding pseudoinverse by correlation processing, to improve efficiency and stability. The simulated data of vibrating point targets and a tank model at $220 \mathrm{GHz}$ will validate the performance of the proposed algorithm.

\section{Models of THz-SAR Vibrating Targets}

As illustrated in Figure 1, the radar moves at velocity $V_{a}$. Then at slow time $t$ it moves to

$$
y^{\prime}=V_{a} t=R_{c} \tan \theta \approx R_{c} \theta .
$$

We could see that $\theta$ has the similar meaning as slow time $t$, and thus the symbol $\theta_{t}$ may be more appropriate; nevertheless it is abandoned for brevity. Now considering an arbitrary moving target, let vector $\boldsymbol{\eta}$ represent the target motion parameters, such as the initial position $(x, y)$, velocity, and vibration frequency. $\boldsymbol{\eta}$ can thus be regarded as the identification code of a target. Suppose that when the radar moves to $y^{\prime}=R_{c} \theta$ or $\theta$ for simplicity, the target of $\boldsymbol{\eta}$ moves to $\left(x_{\eta, \theta}, y_{\eta, \theta}\right)$, and then the target range model is

$$
\begin{aligned}
R_{\boldsymbol{\eta}}(\theta) & =\sqrt{\left(R_{c}+x_{\boldsymbol{\eta}, \theta}\right)^{2}+\left(y^{\prime}+y_{\boldsymbol{\eta}, \theta}\right)^{2}} \\
& \approx \sqrt{R_{c}^{2}+y^{\prime 2}}+x_{\boldsymbol{\eta}, \theta} \cos \theta+y_{\boldsymbol{\eta}, \theta} \sin \theta
\end{aligned}
$$

where the approximation is a result of plane waves; that is, in Figure 1(b) $|P T| \approx\left|P T^{\prime}\right|=|P O|+\left|O T^{\prime}\right|=\sqrt{R_{c}^{2}+y^{\prime 2}}+$ $x_{\eta, \theta} \cos \theta+y_{\eta, \theta} \sin \theta$.

Spotlight SAR echoes of the target could then be represented in the wavenumber domain as

$$
\begin{aligned}
s(K, \theta ; \boldsymbol{\eta})= & P(K) \exp \left[-j K \sqrt{R_{c}^{2}+y^{\prime 2}}\right] \\
& \cdot \exp \left[-j K\left(x_{\boldsymbol{\eta}, \theta} \cos \theta+y_{\boldsymbol{\eta}, \theta} \sin \theta\right)\right],
\end{aligned}
$$

where $K=4 \pi / \lambda=4 \pi f / c$ is the two way wavenumber, $c$ is the speed of light, $\lambda$ is the wavelength, $f$ is the frequency, $K_{C}=4 \pi / \lambda_{C}=4 \pi f_{C} / c$ is the center wavenumber, $\lambda_{C}$ is the carrier wavelength, $f_{C}$ is the carrier frequency, and $P(K)$ is the Fourier transform of the transmitted signal. Suppose multiple targets exit, recall that $\boldsymbol{\eta}$ is the identification code of a target, and thus we use $a(\boldsymbol{\eta})$ as the scattering coefficient of the target with respect to $\boldsymbol{\eta}$. Then the total echoes of all targets can be expressed in terms of integration as

$$
S_{\text {total }}(K, \theta)=\int a(\boldsymbol{\eta}) s(K, \theta ; \boldsymbol{\eta}) \mathrm{d} \boldsymbol{\eta}
$$

In (3), $P(K)$ and $\exp \left[-j K \sqrt{R_{c}^{2}+y^{\prime 2}}\right]$ contain no unknowns and hence they can be compensated for range compression (multiplication by $P^{*}(K)$ ) and motion compensation (multiplication by $\exp \left[j K \sqrt{R_{c}^{2}+y^{\prime 2}}\right]$ ). Thus the first two terms of $s(\cdot)$ in (3) disappear, and then the target signal model becomes

$$
\begin{aligned}
G(K, \theta) \\
\quad=\int a(\boldsymbol{\eta}) \exp \left[-j K\left(x_{\boldsymbol{\eta}, \theta} \cos \theta+y_{\boldsymbol{\eta}, \theta} \sin \theta\right)\right] \mathrm{d} \boldsymbol{\eta} .
\end{aligned}
$$

Note (5) is a Fredholm integral equation of the first kind. When the target experiences vibration, the real vehicle vibration frequencies have complex components and the vibration frequencies also differ for its parts such as the roof and the bumper [12]. However, it can be regarded as a synthesis of simple harmonic vibrations, as measured for two idle cars which can be seen later in the experiment section. Considering only one frequency component for simplicity and without loss of generality, we have

$$
\begin{aligned}
& x_{\eta, \theta}=x+r \cos \left(2 \pi f_{m} t+\varphi_{0}\right), \\
& y_{\eta, \theta}=y+r \sin \left(2 \pi f_{m} t+\varphi_{0}\right),
\end{aligned}
$$

where vibration parameters compose a parameter vector

$$
\boldsymbol{\eta} \stackrel{\text { def }}{=}\left[x, y, r, f_{m}, \varphi_{0}\right]^{T},
$$

and $(x, y)$ is the center of the vibration axis, $r$ is the effective vibration amplitude, $f_{m}$ is the vibration frequency, and $\varphi_{0}$ is the initial phase at $t=0$. By the way, (6) can also be used to model target rotations. 


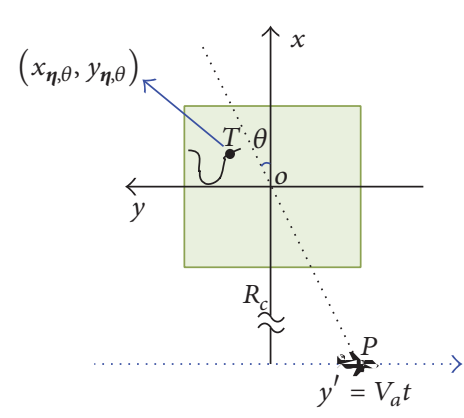

(a)

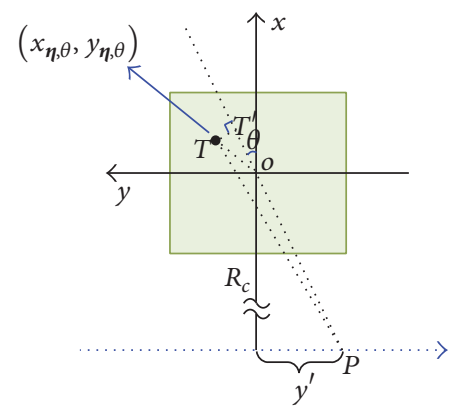

(b)

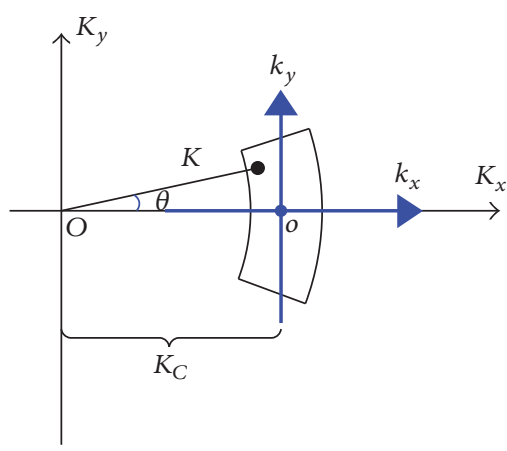

(c)

FIGURE 1: Spotlight SAR moving target geometry: (a) slant plane view; (b) plane wave approximation; (c) wavenumber-domain view.

Substituting (6) into (5), discretizing the result, and adding noise yield

$$
G(K, \theta)=\sum_{i=1}^{I} a_{i} \cdot e_{K \theta}\left(\boldsymbol{\eta}_{i}\right)+v_{i},
$$

where $I$ is the number of vibrating targets (it can also include stationary body points by just setting $r$ or $f_{m}$ to zero and can include no target by setting $a_{i}$ to zero), $a_{i}$ denotes the scattering coefficient of the $i$ th target, and

$$
\begin{aligned}
e_{K \theta}\left(\boldsymbol{\eta}_{i}\right) & \\
\stackrel{\text { def }}{=} & \exp \left(-j K x_{i} \cos \theta-j K y_{i} \sin \theta\right) \\
& \quad \cdot \exp \left(-j K r_{i} \cos \left(\frac{2 \pi f_{m i} R_{c}}{V_{a}} \tan \theta+\varphi_{0 i}\right)\right) .
\end{aligned}
$$

During the derivation of (9), $t=R_{c} \tan \theta / V_{a}$ from (1) and $2 \pi f_{m} R_{c} / V_{a} \gg 1$ have been used.

In (9), we can clearly see that there is an additional exponential component, that is, the last term, representing target vibration in (9) when compared with the stationary scattering center model. Note $K$ and $\theta$ can also be discretized into $M$ and $N$ values, respectively, and therefore (8) can be expressed in a matrix form as

$$
\mathbf{g}=\boldsymbol{\Phi} \mathbf{a}+\mathbf{v}
$$

where

$$
\begin{aligned}
& \mathbf{g}=\left[G\left(K_{1}, \theta_{1}\right), G\left(K_{1}, \theta_{2}\right), \ldots, G\left(K_{M}, \theta_{N}\right)\right]^{T}, \\
& \mathbf{a} \stackrel{\text { def }}{=}\left[a_{1}, a_{2}, \ldots, a_{I}\right]^{T}, \\
& \mathbf{v} \stackrel{\text { def }}{=}\left[v_{1}, v_{2}, \ldots, v_{I}\right]^{T}, \\
& \boldsymbol{\Phi}=\left[\begin{array}{cccc}
e_{K_{1} \theta_{1}}\left(\boldsymbol{\eta}_{1}\right) & e_{K_{1} \theta_{1}}\left(\boldsymbol{\eta}_{2}\right) & \cdots & e_{K_{1} \theta_{1}}\left(\boldsymbol{\eta}_{I}\right) \\
e_{K_{1} \theta_{2}}\left(\boldsymbol{\eta}_{1}\right) & e_{K_{1} \theta_{2}}\left(\boldsymbol{\eta}_{2}\right) & \cdots & e_{K_{R_{1}} \theta_{2}}\left(\boldsymbol{\eta}_{I}\right) \\
\vdots & \vdots & \cdots & \cdots \\
e_{K_{M} \theta_{N}}\left(\boldsymbol{\eta}_{1}\right) & e_{K_{M} \theta_{N}}\left(\boldsymbol{\eta}_{2}\right) & \cdots & e_{K_{M} \theta_{N}}\left(\boldsymbol{\eta}_{I}\right)
\end{array}\right] .
\end{aligned}
$$

From (10), the imaging or scattering coefficient reconstruction problem can be regarded as an inverse problem of solving linear equations.

What deserves special mention is that the unknown $\boldsymbol{\eta}$ has also been discretized into $\boldsymbol{\eta}_{1}, \boldsymbol{\eta}_{2}, \ldots, \boldsymbol{\eta}_{I}$, which are located at a high-dimensional cubic grid, and therefore $\Phi$ contains no unknowns.

\section{The Influence of Target Vibration on THZ-SAR Image}

It is well known that vibrating targets cannot be focused in microwave SAR images and a ghost image will generally result according to the paired echo principle. We now try to analyze THz-SAR image characteristics of vibrating targets. The space between ghosts is a constant [2]

$$
\Delta y=\frac{f_{m} \lambda_{C} R_{c}}{2 V_{a}} .
$$

For THz-SAR, $\Delta y$ will decrease by a commensurate amount compared with microwave SAR since $\lambda_{C}$ is significantly smaller than the wavelength at X-band or $\mathrm{mmW}$ band.

Note the azimuth resolution $\rho_{a}=\lambda_{C} R_{c} / 2 V_{a} T_{l}$, where $T_{l}$ is the coherent processing interval (CPI), and the space in pixel or resolution cell can be expressed as

$$
\frac{\Delta y}{\rho_{a}}=f_{m} T_{l} .
$$

It is apparent that the space in pixel or resolution cell has no relation with the frequency band. However, $T_{l}$ needed for the same resolution as microwave SAR is reduced for THzSAR. For typical parameter values, $\Delta y / \rho_{a} \leq 1$, which states that the ghosts are not resolvable and line segment results.

According to Carson's rule [22], the ghost number plus the real target is

$$
N \approx \frac{8 \pi r}{\lambda_{C}}+3 .
$$

From (12) and (14), the length of the ghost image or the line segment in azimuth is

$$
\Delta L=\Delta y N=\frac{\left(8 \pi r+3 \lambda_{C}\right) f_{m} R_{c}}{2 V_{a}} .
$$


Equation (15) makes it clear that $\Delta L$ will decrease when the frequency moves to the terahertz band.

\section{Vibrating Target Imaging via the Improved ExCoV Method}

In order to solve (8), we model the pdf of THz-SAR echo vector $\mathbf{g}$ given a and $\sigma^{2}$ using the standard additive Gaussian noise model

$$
p\left(\mathbf{g} \mid \mathbf{a}, \sigma^{2}\right)=N\left(\mathbf{g} ; \boldsymbol{\Phi} \mathbf{a}, \sigma^{2} \mathbf{C}\right),
$$

where $\mathbf{C}$ is a known positive definite symmetric matrix of size $M N \times M N, \sigma^{2}$ is an unknown noise-variance parameter, and $\sigma^{2} \mathbf{C}$ is the noise covariance matrix. The SBL employs a Gaussian prior on the signal with a distinct variance component on each signal element

$$
\begin{aligned}
p\left(a_{i} ; \gamma_{i}\right)=N\left(0, \gamma_{i}\right)=\left(2 \pi \gamma_{i}\right)^{-1 / 2} \exp \left(-\frac{a_{i}^{2}}{2 \gamma_{i}}\right), & \\
& i=1, \ldots, I .
\end{aligned}
$$

However, we have known, according to the sparse recovery theory and a priori knowledge, that in most real-world cases only a few elements of a have significant magnitudes and that the remaining elements are either strictly zeros or close to zeros [23]. Therefore the prior distribution (17) did not capture the signal key feature, that is, sparsity. On the contrary, the ExCoV method assigns distinct variance components to the candidates for significant signal elements and uses only one common variance-component parameter to account for the variability of the rest of signal coefficients [24]. Let $\Lambda$ be the full index set in the signal and let the size be I. Denote $\mathbf{A}$ as the set of indices of the signal elements with distinct variance components and the size as $m_{\mathbf{A}}$, and define the complementary index set $\mathbf{B}=\boldsymbol{\Lambda} / \mathbf{A}$ with cardinality $m_{\mathbf{B}}=$ $I-m_{\mathbf{A}}$. Accordingly, we partition $\boldsymbol{\Phi}$ and $\mathbf{a}$ into submatrices $\boldsymbol{\Phi}_{\mathrm{A}}$ and $\boldsymbol{\Phi}_{\mathrm{B}}$ and subvectors $\mathbf{a}_{\mathrm{A}}$ and $\mathbf{a}_{\mathrm{B}}$. Then the following prior model for the signal coefficients is adopted:

$$
\begin{aligned}
p\left(\mathbf{a} \mid \boldsymbol{\delta}_{\mathbf{A}}, \gamma^{2}\right)= & p\left(\mathbf{a}_{\mathbf{A}} \mid \boldsymbol{\delta}_{\mathbf{A}}\right) \cdot p\left(\mathbf{a}_{\mathbf{B}} \mid \gamma^{2}\right) \\
= & N\left(\mathbf{a}_{\mathbf{A}} ; 0_{m_{\mathbf{A}} \times 1}, D_{\mathbf{A}}\left(\boldsymbol{\delta}_{\mathbf{A}}\right)\right) \\
& \cdot N\left(\mathbf{a}_{\mathbf{B}} ; 0_{m_{\mathbf{B}} \times 1}, D_{\mathbf{B}}\left(\gamma^{2}\right)\right),
\end{aligned}
$$

where $D_{\mathbf{A}}\left(\boldsymbol{\delta}_{\mathbf{A}}\right)=\operatorname{diag}\left\{\delta_{\mathbf{A}, 1}^{2}, \delta_{\mathbf{A}, 2}^{2}, \ldots, \delta_{\mathbf{A}, m_{\mathbf{A}}}^{2}\right\}$ and $D_{\mathbf{B}}\left(\gamma^{2}\right)=$ $\gamma^{2} \mathbf{I}_{m_{\mathrm{B}}}$ are the signal covariance matrices and the variance components $\delta_{\mathbf{A}, 1}^{2}, \delta_{\mathbf{A}, 2}^{2}, \ldots, \delta_{\mathbf{A}, m_{\mathbf{A}}}^{2}$ for $\mathbf{a}_{\mathbf{A}}$ are distinct while the common variance $\gamma^{2}$ accounts for the variability of $\mathbf{a}_{\mathbf{B}}$. Assume that the signal variance components $\boldsymbol{\delta}_{\mathbf{A}}$ and $\gamma^{2}$ are unknown and define the set of all unknowns $\boldsymbol{\theta}=$ $\left(\mathbf{A}, \boldsymbol{\delta}_{\mathbf{A}}, \gamma^{2}, \sigma^{2}\right)$, and then the marginal pdf of the observations g given $\boldsymbol{\theta}$ is [24]

$$
\begin{aligned}
p(\mathbf{g} \mid \boldsymbol{\theta}) & =\int p\left(\mathbf{g} \mid \mathbf{a}, \sigma^{2}\right) \cdot p\left(\mathbf{a} \mid \boldsymbol{\delta}_{\mathbf{A}}, \gamma^{2}\right) \mathrm{d} \mathbf{a} \\
& =N\left(\mathbf{g} ; 0_{M N \times 1}, P^{-1}(\boldsymbol{\theta})\right),
\end{aligned}
$$

where $P(\boldsymbol{\theta})$ is the inverse covariance matrix of $\mathbf{g}$ given $\boldsymbol{\theta}$. Clearly, $p(\mathbf{g} \mid \boldsymbol{\theta})$ provides generalized maximum-likelihood (GML) estimation of $\boldsymbol{\theta}$. The GML estimation of $p(\mathbf{g} \mid$ a, $\left.\sigma^{2}\right) p\left(\mathbf{a} \mid \boldsymbol{\delta}_{\mathbf{A}}, \gamma^{2}\right)$ proceeds for obtaining the only unknown a as the image. The whole estimation process is iteratively realized via applying the ExCoV algorithm, and the iteration will terminate when $\mathbf{A}$ does not change between two consecutive cycles [24].

ExCoV has demonstrated an excellent algorithm for solving linear equations arising from the real world especially from the radar field [25]. Compared to SBL (SBL-EM, SBLType II, VBSBL, and ESBL), SpaRSA, and CoSaMP, it has the smallest mean-square error (MSE) in the low signal-to-noise ratio (SNR) and the second highest computation efficiency (not better than CoSaMP whose MSE is large). It begins with an initialization followed by complex iterations. The initialization provides the starting point for the following iterations and thus plays an important part for fast convergence. The original ExCoV utilizes Moore-Penrose pseudoinverse to initialize the parameters; that is,

$$
\mathbf{a}^{(0)}=\Phi^{H}\left(\Phi \Phi^{H}\right)^{-1} \mathbf{g}
$$

where $H$ denotes complex conjugation. The first $m_{\mathbf{A}}^{(0)}$ largest elements of $\mathbf{a}^{(0)}$ are found to initialize $\mathbf{A}$.

However, $\boldsymbol{\Phi} \boldsymbol{\Phi}^{H}$ is usually rank deficient for real radar problems and hence $\boldsymbol{\Phi} \boldsymbol{\Phi}^{H}$ is not inversible, resulting in wrong estimation of $\mathbf{a}^{(0)}$. An example is shown in Figure 2(a). We recognize that $\boldsymbol{\Phi} \Phi^{H}$ denotes autocorrelation and crosscorrelation of the measurement matrix and will give a sincshaped system response. $\left(\boldsymbol{\Phi} \boldsymbol{\Phi}^{H}\right)^{-1}$ is just the inverse sinc response, which is an identity matrix in ideal condition, to realize super-resolution estimation of a. Thus we abandon this term and obtain a more robust, efficient, and simpler estimation than (20) as

$$
\mathbf{a}^{(0)}=\Phi^{H} \mathbf{g}
$$

which can also be called correlation imaging, back projection imaging, or multidimensional matched filtering imaging. The estimation is displayed in Figure 2(b).

\section{Experiment Results}

5.1. Vibration Measurement Results of Two Idle Cars. We at first measured the vibration of two cars, that is, a Mengshi car and a Nissan car, whose engines were running idly, by a frequency-modulated continuous wave (FMCW) radar, as Figures 3(a) and 3(b) show. The carrier frequency is $25 \mathrm{GHz}$ and the bandwidth is $2 \mathrm{GHz}$ which results in a $7.5 \mathrm{~cm}$ resolution in range. The phase $\varphi(t)$ is measured and therefore the vibration range $r$ can be obtained by $r(t)=\varphi(t)(c / 4 \pi)$ after the phase filtering and the DC being eliminated, as shown in Figure 3(c). Finally, the vibration spectra can be obtained simply by the Fourier transform of $r(t)$, as displayed in Figure 3(d). From the spectra, it can clearly be seen that only few strong vibration frequency components, about 2 3 for our subjects, exist for idle cars. Therefore, one can model 


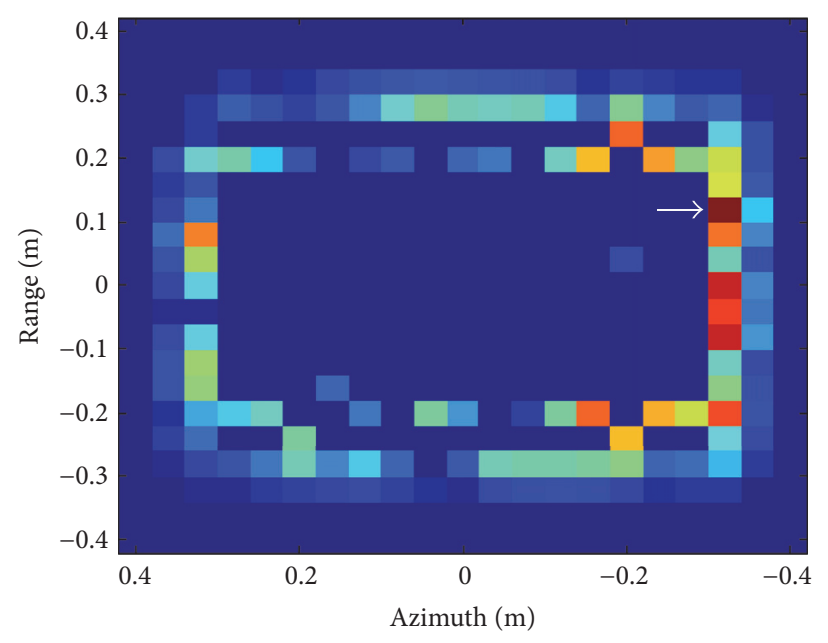

(a)

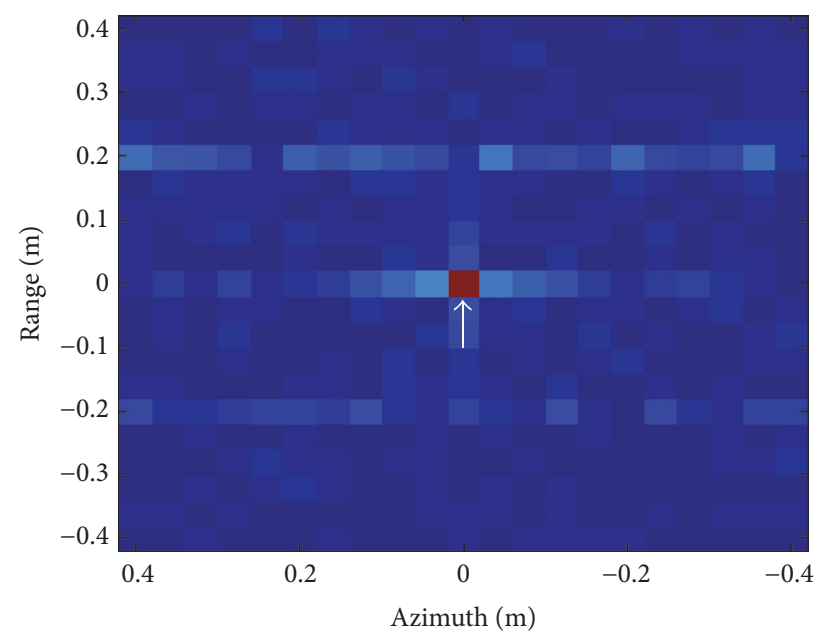

(b)

FIGURE 2: Initial imaging of a vibrating target at $(0,0)$ by different methods (the arrow points to the maxima and the true maximum is located at $(0,0))$ : (a) Moore-Penrose pseudoinverse and (b) correlation.

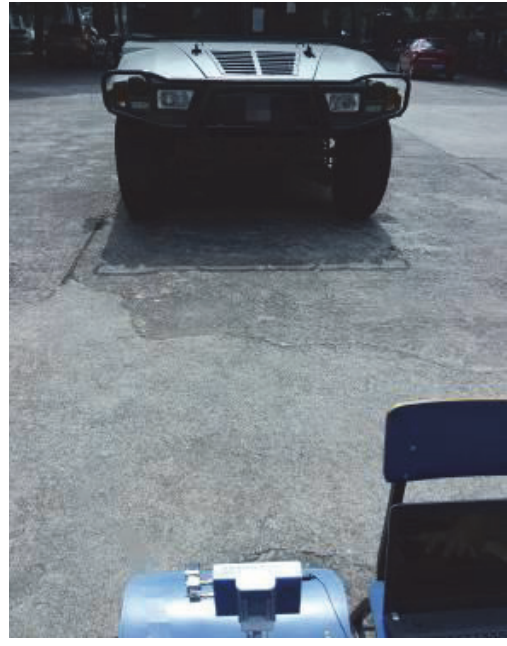

(a)

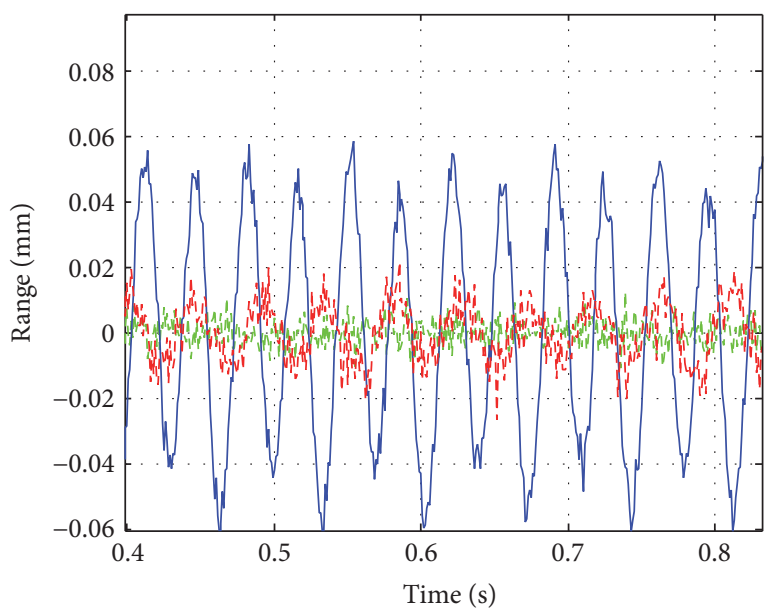

Stationary Mengshi car

- Idle Mengshi car
- - Idle Nissan car

(c)

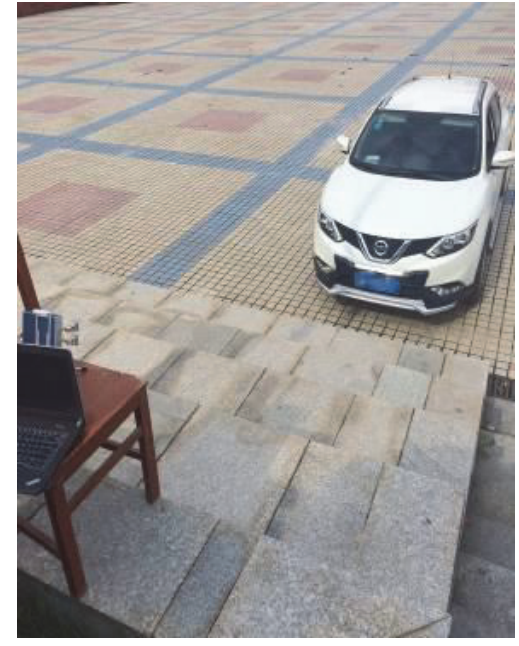

(b)

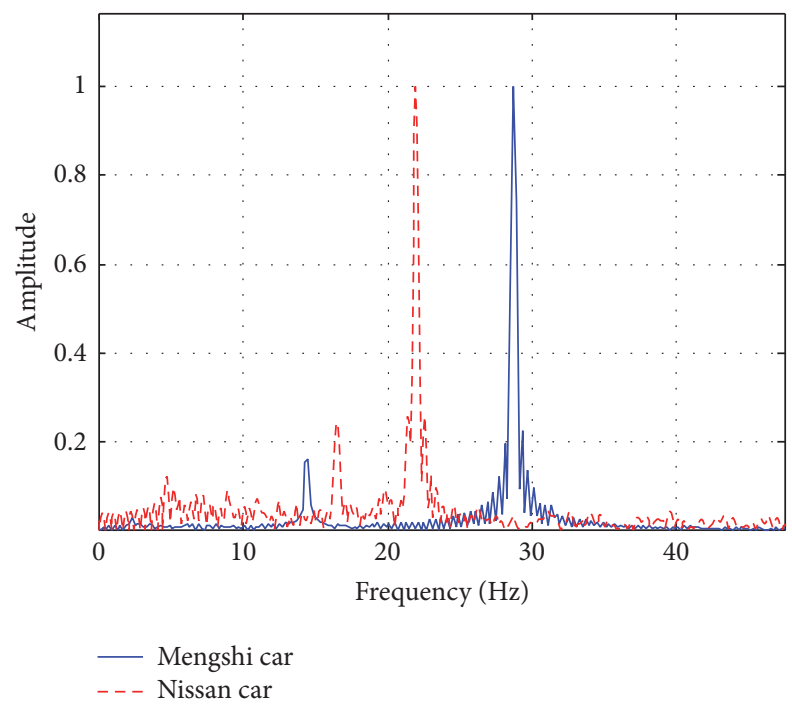

(d)

FIGURE 3: Vibration measurement results of two idle cars: (a) Mengshi car; (b) Nissan car; (c) vibration amplitude versus time; (d) vibration spectra. 


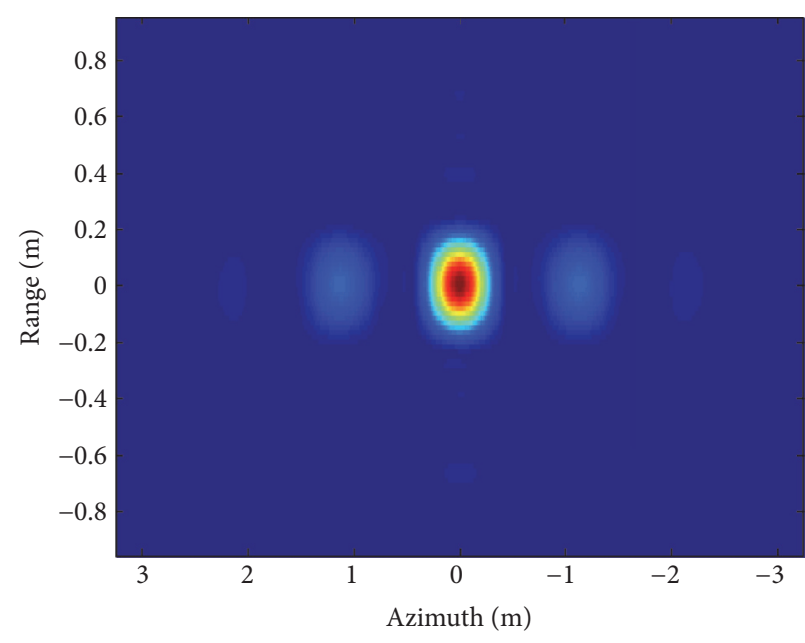

(a)

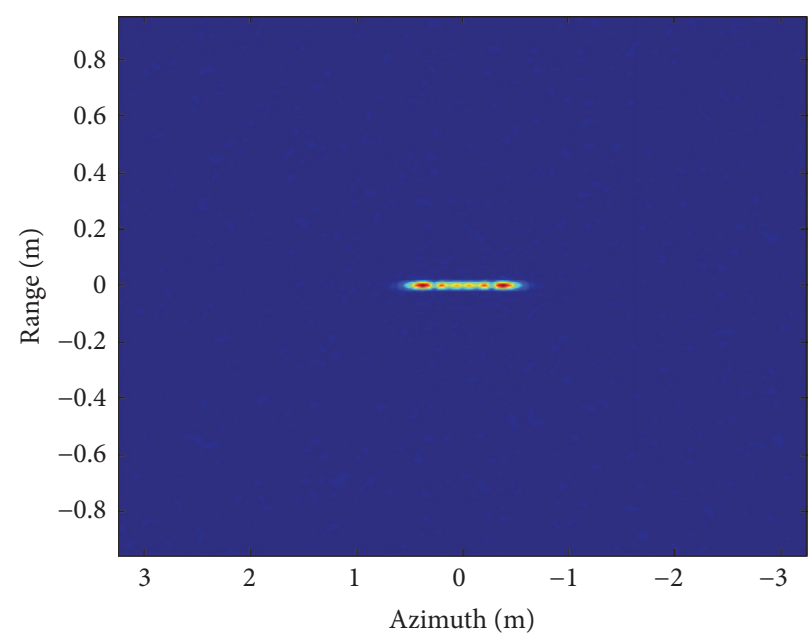

(b)

FIGURE 4: SAR images of a vibrating point target with the vibration amplitude $1 \mathrm{~mm}$ and frequency $1 \mathrm{~Hz}$ without refocusing: (a) X-band; (b) THz band (220 GHz).

the vibration by the synthesis of simple harmonic vibrations. In the paper, we only use one component for simplicity and without loss of generality.

\subsection{THz-SAR Imaging Results of Simulated Vibrating Targets.} We proceed to conduct the following numerical experiment, as real experiments are right now not available due to low power of our $\mathrm{THz}$ source available, to validate the algorithm proposed. THz-SAR system parameters are given in Table 1. The experiment includes comparison of vibrating target images in microwave and terahertz bands and imaging of vibrating point targets as well as a tank model.

5.2.1. Comparison of Vibrating Target Images in Microwave and Terahertz Bands. Figure 4 provides a good representation of original image characteristics of a vibrating target in different bands. For the X-band, the resolution is $0.15 \mathrm{~m}$ and $0.28 \mathrm{~m}$ in range and azimuth, respectively. Only a few ghosts are present in the image as Figure 4(a) shows, and the ghosts bear strong resemblance to stationary point targets or their sidelobes, hence difficult to discriminate between vibration and stillness. In contrast, the vibrating target takes on a line segment in the THz-SAR image as Figure 4(b) depicts. Clearly, terahertz can enforce the vibrating target image feature and therefore is expected to be used as the best band for vibration sensing. In addition, the line segment in Figure 4(b) also consists of ghosts, while the spacing between which is so near in the terahertz regime that is not resolved, as (13) predicts.

5.2.2. Vibrating Point Target Imaging. For quantitatively analyzing the performance of the algorithm proposed, we use three point targets in the experiment herein, whose parameters are shown in Table 1 . The images formulated by the polar format algorithm (PFA) are displayed in Figure 5, where the stationary target is well focused at the bottom while the two vibrating ones are blurred into segments.

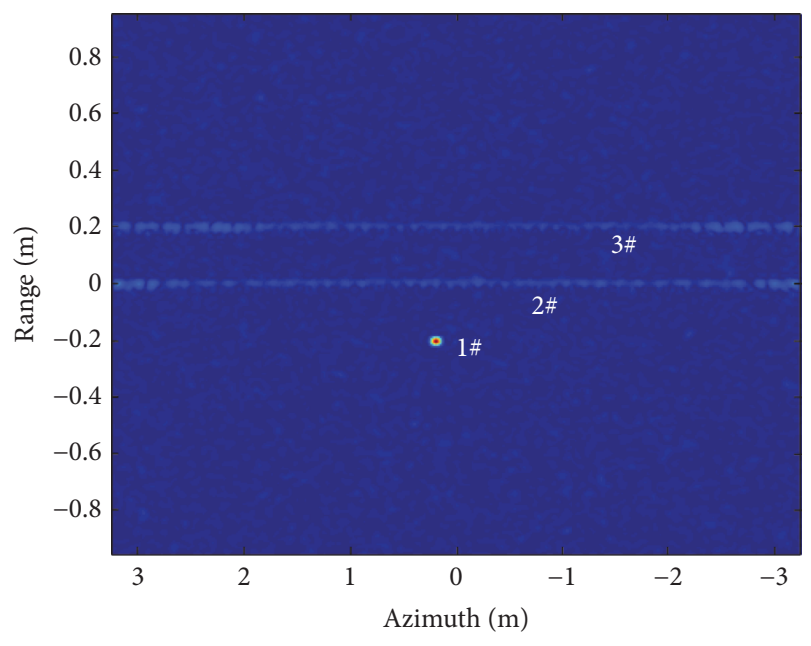

FIgURE 5: THz-SAR image of point targets in Table 2, without refocusing.

TABLE 1: THz-SAR parameters.

\begin{tabular}{lccc}
\hline Mode & Spotlight & CPI & $0.5 \mathrm{~s}$ \\
\hline Carrier frequency & $220 \mathrm{GHz}$ & Bandwidth & $10 \mathrm{GHz}$ \\
Slant angle & $0^{\circ}$ & Platform velocity & $80 \mathrm{~m} / \mathrm{s}$ \\
Range resolution & $0.015 \mathrm{~m}$ & Azimuth resolution & $0.051 \mathrm{~m}$ \\
\hline
\end{tabular}

Now we employ the correlation method and the improved ExCoV to reconstruct vibrating target scattering coefficients, hence obtaining their refocused images. The imaged size is just set $0.8 \mathrm{~m} \times 0.8 \mathrm{~m}$ both with $0.04 \mathrm{~m}$ grid spacing for the memory as well as computation time costs, and the sparse sampling rate is $40 \%$, implying that compressive sensing is utilized together with ExCoV. Multidimensional images are obtained with the dimension in accord with $\eta \stackrel{\text { def }}{=}\left[x, y, r, f_{m}, \varphi_{0}\right]^{T}$, and parts of their slices along the 

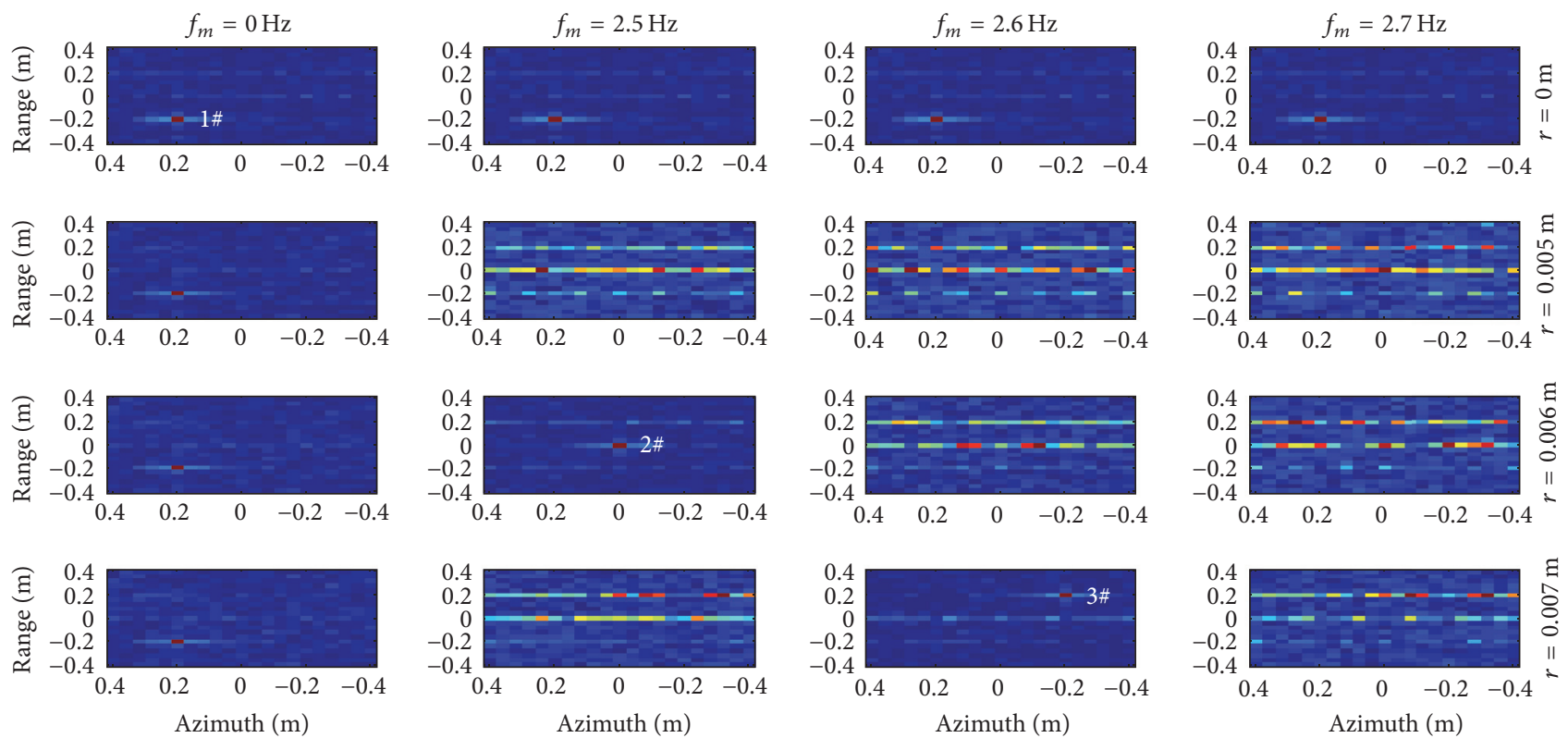

FIgURE 6: THz-SAR image of point targets in Table 2 under $-10 \mathrm{~dB}$ SNR, refocused via correlation imaging.
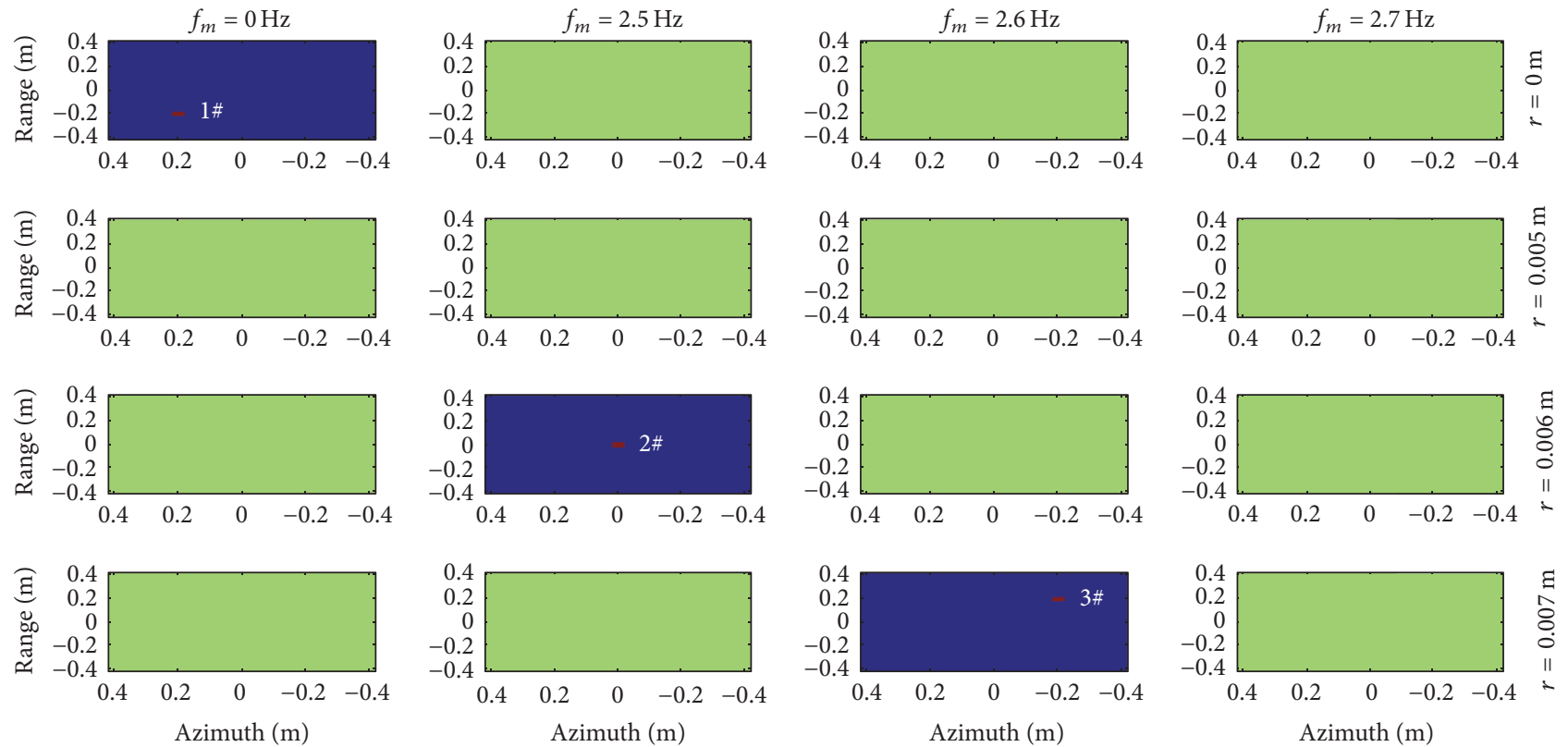

FIgURE 7: THz-SAR image of point targets in Table 2 under -10 dB SNR, refocused via ExCoV imaging.

vibration frequency $f_{m}$ and amplitude $r$ are shown in Figures 6 and 7. Clearly, the ExCoV method provides better imaging results, while the image by the correlation method shows high sidelobes and generates some artifacts for the stationary target. From the location of the focused image slices in Figure 7, one can readily estimate the vibration parameters.

We define the root MSE (RMSE) between the reconstructed scattering coefficient and the true values, that is, $\mathrm{RMSE}=\|\widetilde{\mathbf{a}}-\widehat{\mathbf{a}}\| /\|\widetilde{\mathbf{a}}\|$, where $\tilde{\mathbf{a}}$ denotes the true values, to test the algorithm performance. 100 times Monte Carlo simulations are conducted for each SNR value to obtain Figure 8, indicating that RMSE drops sharply with SNR.
Excellent performance can be realized in low SNR and Figure 7 is an example of $-10 \mathrm{~dB}$ SNR.

5.2.3. Vibrating Tank Model Imaging. A real SAR image around the Isleta Lake in New Mexico (Figure 9(b)) is downloaded from the Sandia Cooperation website and is used to generate the scene echoes [26]. Then a simulated vibrating tank model (Figure $9(\mathrm{a})$ ) is fed into the scene with the size $0.37 \mathrm{~m} \times 0.2 \mathrm{~m} \times 0.18 \mathrm{~m}$ (length by width by height) and with the vibration frequency $2.5 \mathrm{~Hz}$ and amplitude $0.005 \mathrm{~m}$. Its images, when stationary and vibrating, are shown in Figures 9(c) and 9(d). The refocused image slice 


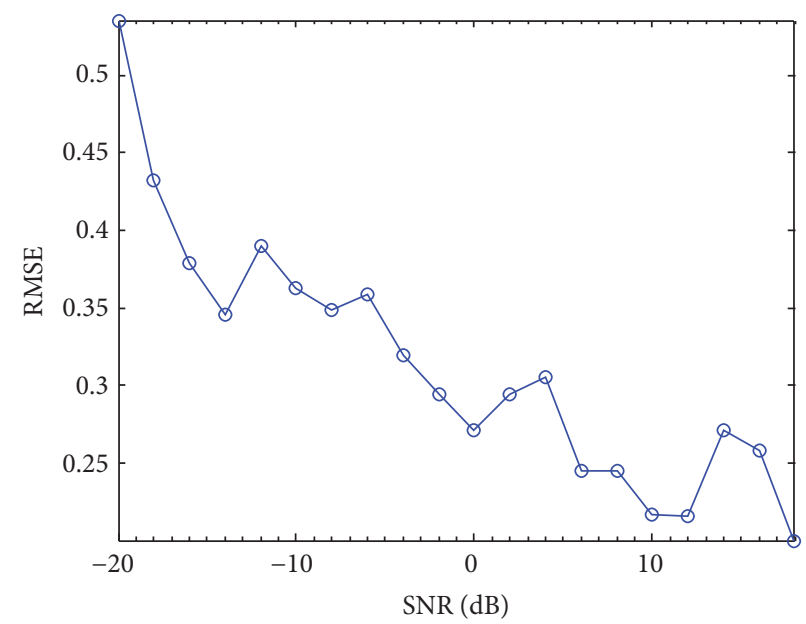

FIgURE 8: The RMSE versus SNR for the ExCoV method.

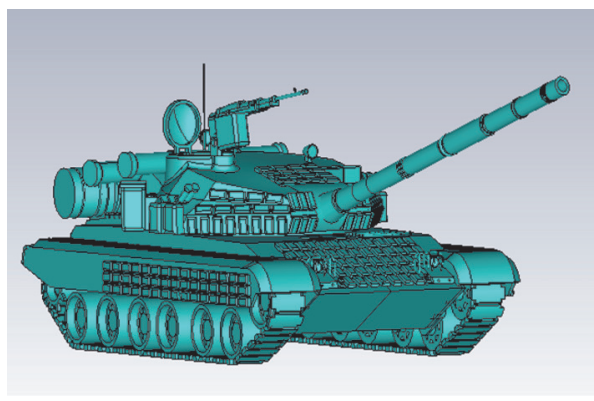

(a)

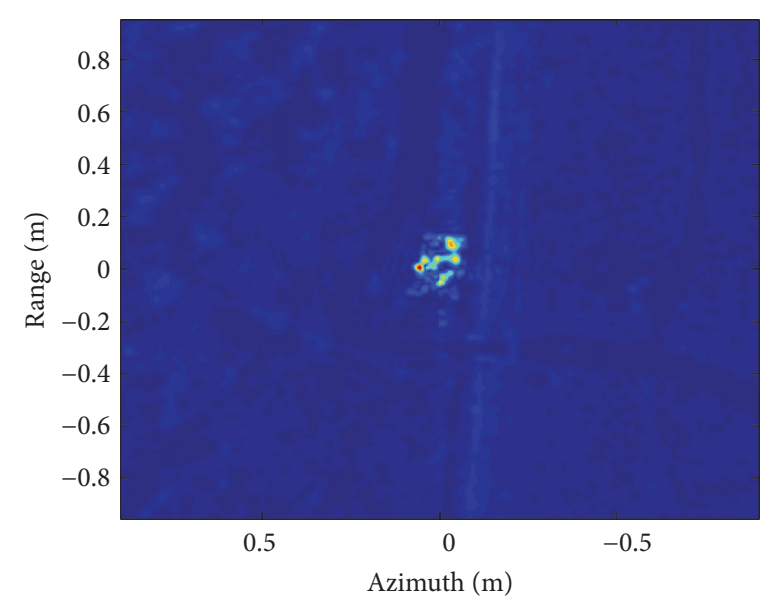

(c)

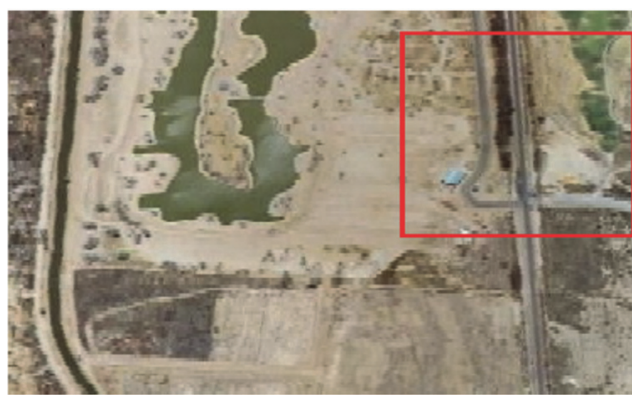

(b)

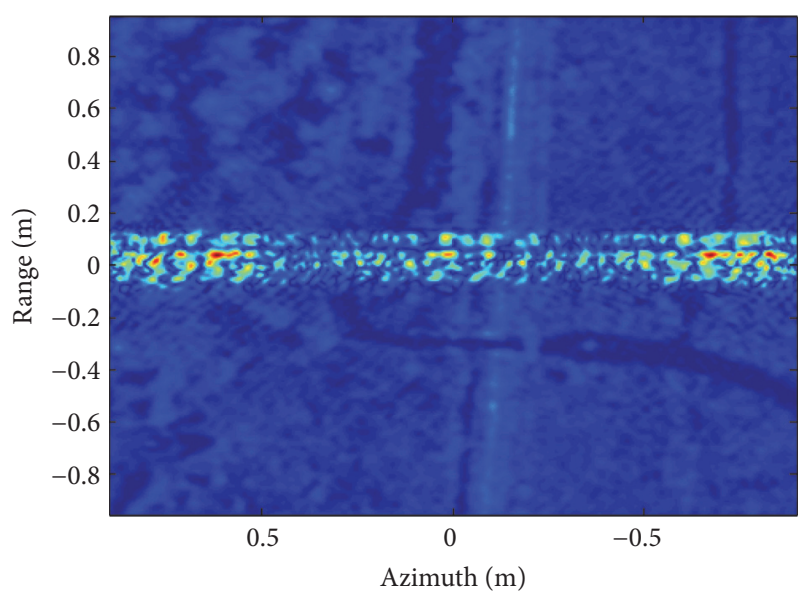

(d)

FIGURE 9: Simulated THz-SAR images of the tank model when stationary and vibrating, respectively. (a) Tank model; (b) Isleta Lake in New Mexico used as the background; (c) image of stationary tank model; (d) image of vibrating tank model.

TABle 2: Point target parameters.

\begin{tabular}{lcccc}
\hline Target number & Azimuth & Range & Vibration frequency & Vibration amplitude \\
\hline 1\# (stationary) & $0.2 \mathrm{~m}$ & $-0.2 \mathrm{~m}$ & 0 & 0 \\
2\# (vibrating) & 0 & 0 & $2.5 \mathrm{~Hz}$ & $0.006 \mathrm{~m}$ \\
3\# (vibrating) & $-0.2 \mathrm{~m}$ & $0.2 \mathrm{~m}$ & $2.6 \mathrm{~Hz}$ & $0.007 \mathrm{~m}$ \\
\hline
\end{tabular}




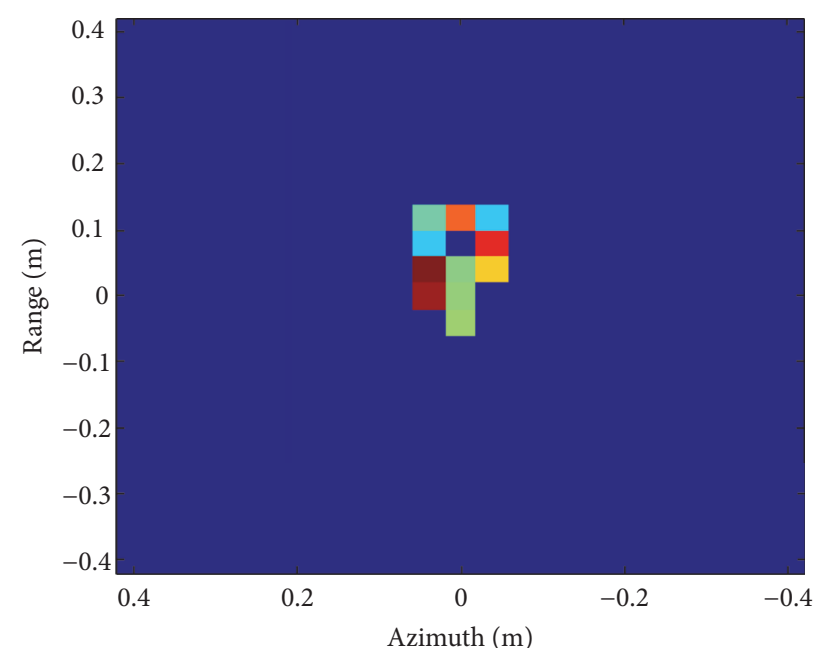

FIGURE 10: THz-SAR image slice of the vibrating tank model on the Isleta Lake background, refocused by the ExCoV method.

is displayed in Figure 10, and the location of the slice indicates the correct vibration frequency and amplitude. In reality, the vibrating parameters will take on estimation error due to parameter grid mismatch, which remains to be explored in future investigations.

\section{Conclusion}

Terahertz has significant advantages of remote vibration sensing over microwave bands. In this paper, the THz-SAR echo model of vibrating targets has been established. Their imaging characteristics with THz-SAR are analyzed and the main features of vibrating point targets are segments consisting of unresolvable ghosts. An improved algorithm based on ExCoV, with more robust and efficient initialization, has been proposed in the sparse Bayesian framework for reconstructing scattering coefficients. Data of vibrating targets and a tank model in a real background has been simulated at $220 \mathrm{GHz}$ and the reconstructed images show good performance at low SNR. The algorithm proposed can also overcome the deficiencies of sidelobes and artifacts arising from traditional correlation (or back projection) imaging method, as compared in Figures 6 and 7. In fact, the algorithm begins with correlation and can be treated as combination of ExCoV and correlation. Terahertz, together with the sparse Bayesian theory, shows a viable and most promising alternative to remote sensing of minor vibrations in particular with rapidly evolving of solid-state and high-power electric-vacuum terahertz devices. Although the Bayesian method provides a fine solution to vibrating target imaging, algorithms with higher efficiency to realize video-rate THz-SAR imaging are still to be studied in future, especially when multiple vibration components are taken into account.

\section{Competing Interests}

The authors declare that they have no competing interests.

\section{Acknowledgments}

The paper is supported by the National Natural Science Foundation for Young Scientists of China under Grants 61302148 and 61571011.

\section{References}

[1] S. Baumgartner, G. Krieger, K. Bethke, and S. Zuev, "Traffic monitoring with SAR: implications of target acceleration," in Proceedings of the European Conference on Synthetic Aperture Radar (EUSAR '06), May 2006.

[2] X. Li, B. Deng, Y. Qin, H. Wang, and Y. Li, "The influence of target micromotion on SAR and GMTI," IEEE Transactions on Geoscience and Remote Sensing, vol. 49, no. 7, pp. 2738-2751, 2011.

[3] E. F. Stockburger and D. N. Held, "Interferometric moving ground target imaging," in Proceedings of the IEEE 1995 International Radar Conference, pp. 438-443, Alexandria, Va, USA, 1995.

[4] B. Deng, G. Wu, Y. Qin, H. Wang, and X. Li, "SAR/MMTI: An extension to conventional SAR/GMTI and a combination of SAR and micromotion techniques," in Proceedings of the IET International Radar Conference, pp. 42-45, Guilin, China, April 2009.

[5] B. Deng, Y. Qin, H. Wang, and Y. Li, "Angular extent effect of micromotion target in SAR image by polar format algorithm," Journal of Systems Engineering and Electronics, vol. 25, no. 3, Article ID 6850221, pp. 428-433, 2014.

[6] B. Deng, H.-Q. Wang, Y.-L. Qin, S. Zhu, and X. Li, "Rotating parabolic-reflector antenna target in SAR data: model, characteristics, and parameter estimation," International Journal of Antennas and Propagation, vol. 2013, Article ID 583865, 13 pages, 2013.

[7] D. B. André, D. Blacknell, D. G. Muff, and M. R. Nottingham, "The physics of vibrating scatterers in SAR imagery," in SPIE Proceedings of the Algorithms for Synthetic Aperture Radar Imagery XVIII, SPIE, Orlando, Fla, USA, April 2011.

[8] B. Deng, H.-Q. Wang, X. Li, Y.-L. Qin, and J.-T. Wang, "Generalised likelihood ratio test detector for micro-motion targets in synthetic aperture radar raw signals," IET Radar, Sonar and Navigation, vol. 5, no. 5, pp. 528-535, 2011.

[9] W. G. Carrara, R. S. Goodman, and R. M. Majewski, Spotlight Synthetic Aperture Radar: Signal Processing Algorithms, Artech House, Norwood, Mass, USA, 1995.

[10] N. S. Subotic, B. J. Thelen, and D. A. Carrara, "Cyclostationary signal models for the detection and characterization of vibrating objects in SAR data," in Proceedings of the Conference Record of the 32nd Asilomar Conference on Signals, Systems \& Computers (ACSSC '98), vol. 2, pp. 1304-1308, November 1998.

[11] Y. Chen, B. Deng, H. Wang, Y. Qin, and J. Ding, "Vibration target detection and vibration parameters estimation based on the DPCA technique in dual-channel SAR," Science China. Information Sciences, vol. 55, no. 10, pp. 2281-2291, 2012.

[12] M. Rüegg, E. Meier, and D. Nüesch, "Vibration and rotation in millimeter-wave SAR," IEEE Transactions on Geoscience and Remote Sensing, vol. 45, no. 2, pp. 293-304, 2007.

[13] W. Zhang, C. Tong, Q. Zhang, Y. Zhang, and X. Zhang, "Extraction of vibrating features with dual-channel fixedreceiver bistatic SAR," IEEE Geoscience and Remote Sensing Letters, vol. 9, no. 3, pp. 507-511, 2012. 
[14] W. Zhang, C. Tong, L. Ren, X. Chen, and X. Zhang, "Bistatic micro-Doppler radar signatures of vibrating targets with alongtrack interferometry," in Proceedings of the 4th IEEE International Symposium on Microwave, Antenna, Propagation and EMC Technologies for Wireless Communications (MAPE '11), pp. 505-508, Beijing, China, November 2011.

[15] Q. Wang, M. Pepin, A. Wright et al., "Reduction of vibrationinduced artifacts in synthetic aperture radar imagery," IEEE Transactions on Geoscience and Remote Sensing, vol. 52, no. 6, pp. 3063-3073, 2014.

[16] B. Deng, Y.-L. Qin, H.-Q. Wang, X. Li, and H. Li, "Pulserepetition-interval transform-based vibrating target detection and estimation in synthetic aperture radar," IET Signal Processing, vol. 6, no. 6, pp. 551-558, 2012.

[17] T. Sparr and B. Krane, "Micro-Doppler analysis of vibrating targets in SAR," IEE Proceedings-Radar, Sonar and Navigation, vol. 150, no. 4, pp. 277-283, 2003.

[18] Q. Wang, M. Pepin, R. J. Beach et al., "SAR-based vibration estimation using the discrete fractional Fourier transform," IEEE Transactions on Geoscience and Remote Sensing, vol. 50, no. 10, pp. 4145-4156, 2012.

[19] Y. Zhang, J. Sun, P. Lei, and W. Hong, "SAR-based paired echo focusing and suppression of vibrating targets," IEEE Transactions on Geoscience and Remote Sensing, vol. 52, no. 12, pp. 7593-7605, 2014.

[20] Y. Qin, B. Deng, Z. Huang, and W. Su, "Hybrid micromotionscattering center model for synthetic aperture radar micromotion target imaging," Journal of Systems Engineering and Electronics, vol. 24, no. 6, pp. 931-937, 2013.

[21] H. B. Wallace, "Video synthetic aperture radar (ViSAR)," DARPA, 2012.

[22] R. G. Wiley, The Interception and Analysis of Radar Signals, Artch House, Bristol, UK, 2006.

[23] J. Ender, "A brief review of compressive sensing applied to radar," in Proceedings of the 14th International Radar Symposium (IRS '13), pp. 3-16, Dresden, Germany, June 2013.

[24] K. Qiu and A. Dogandzic, "Variance-component based sparse signal reconstruction and model selection," IEEE Transactions on Signal Processing, vol. 58, no. 6, pp. 2935-2952, 2010.

[25] W. G. Su, H. Q. Wang, B. Deng, and R. J. Wang, "Sparse Bayesian SAR imaging of moving target via the excov method," in Proceedings of the IEEE Workshop on Statistical Signal Processing, pp. 448-451, June-July 2014.

[26] B. Deng, Y. Qin, Y. Li, H. Wang, and X. Li, "Fast simulation of raw signals from natural scenes for squint stripmap SAR," in Proceedings of the Asia-Pacific Conference on Synthetic Aperture Radar (APSAR '09), pp. 235-238, IEEE, Xian, China, October 2009. 


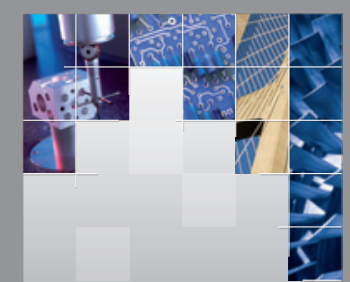

\section{Enfincering}
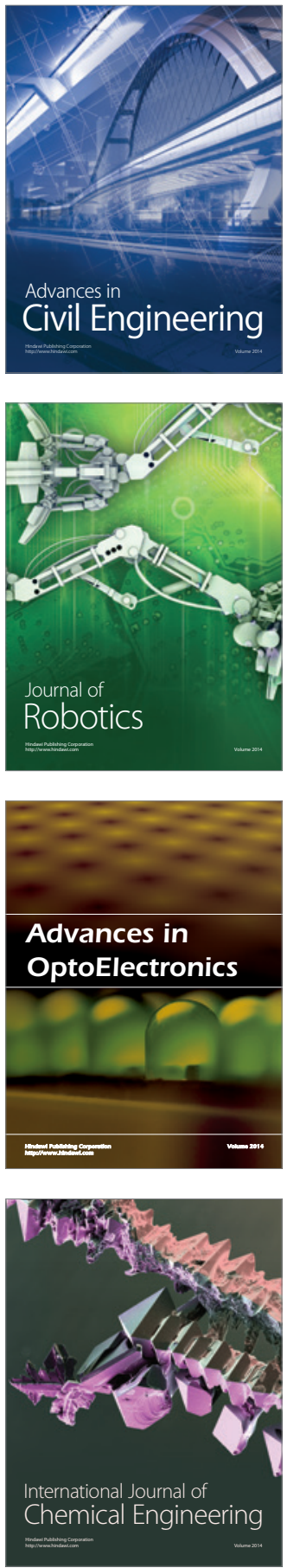

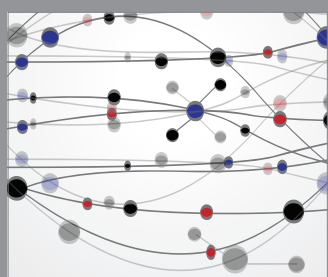

The Scientific World Journal

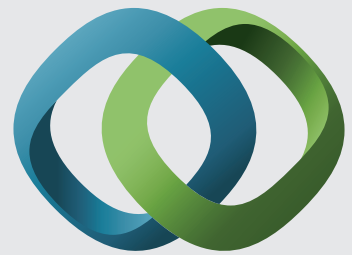

\section{Hindawi}

Submit your manuscripts at

https://www.hindawi.com
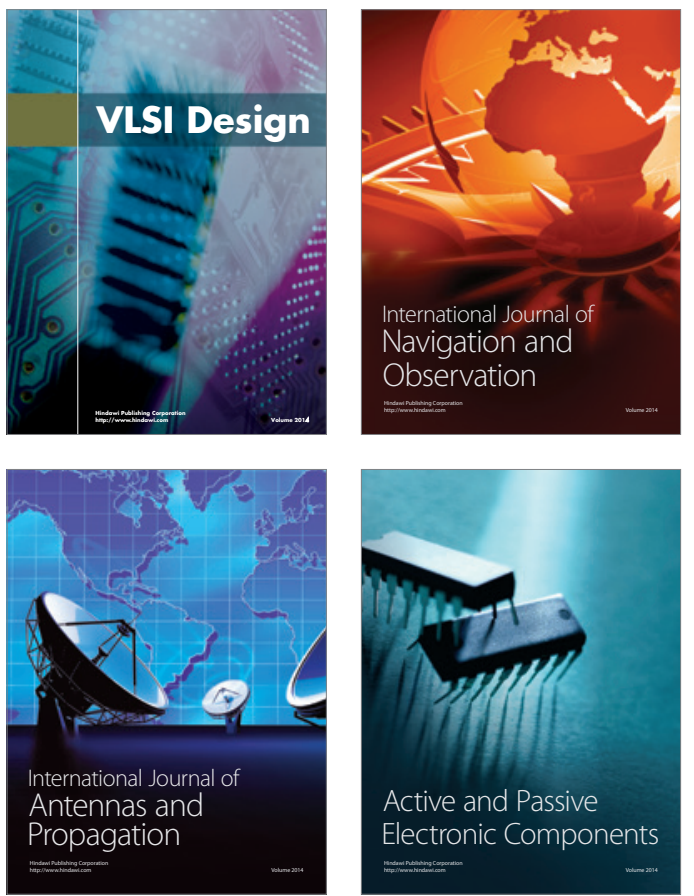
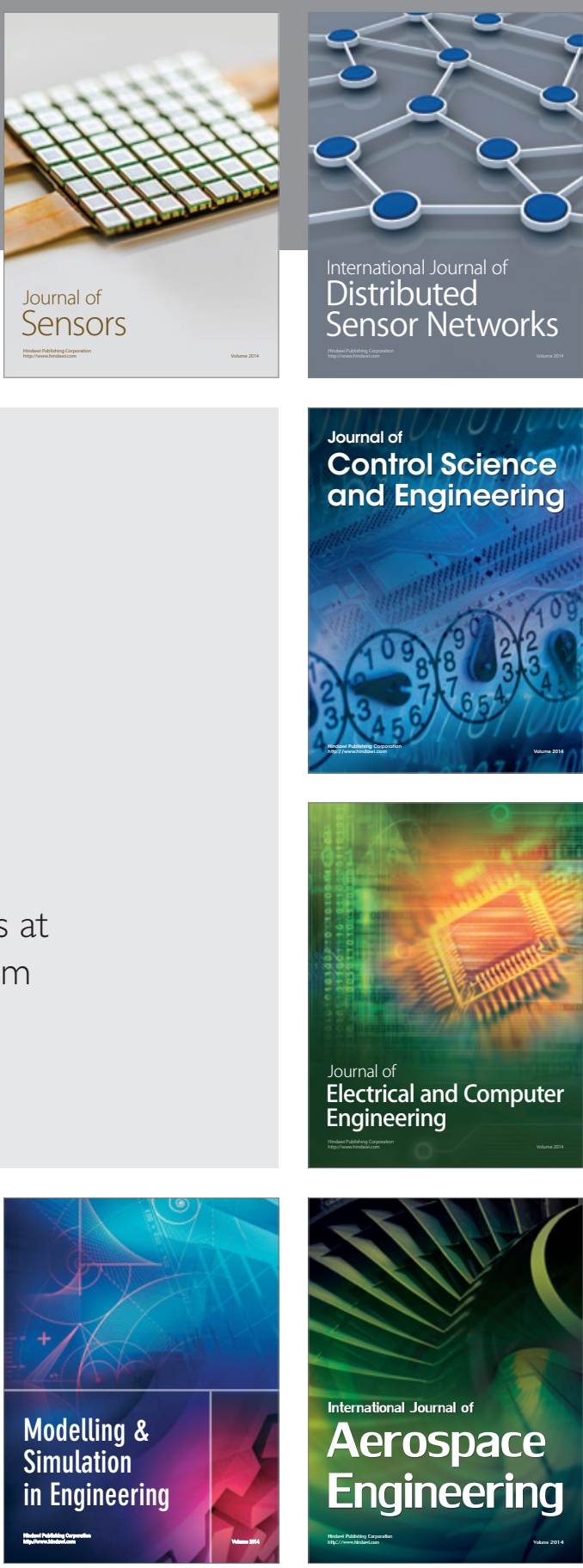

International Journal of

Distributed

Sensor Networks

$-$

Joumal of

Control Science

and Engineering
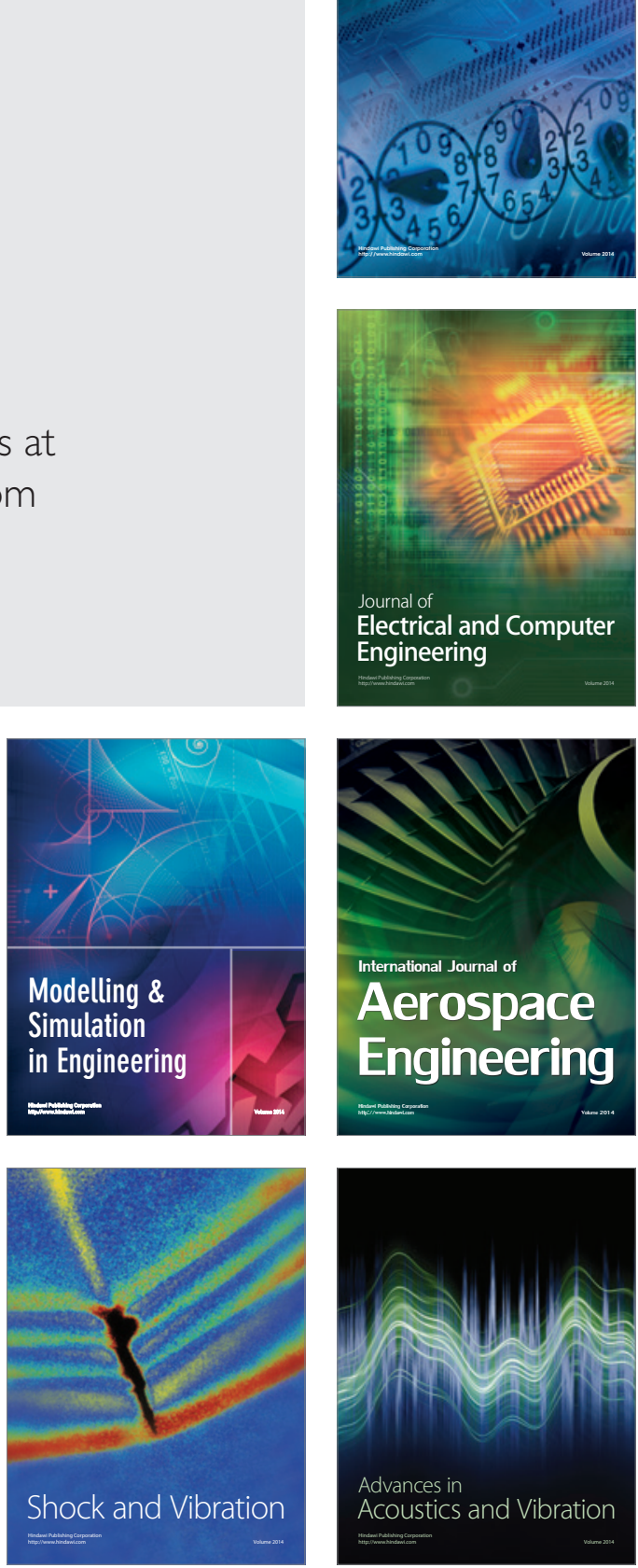